\title{
Epigenetics of Skin Intrinsic Florescence
}

\author{
Jiamin Guo $^{1}$, Andrew Paterson ${ }^{2}$, Delnaz Roshandel ${ }^{2}$ \\ ${ }^{1}$ Biostatistics Division, Dalla Lana School of Public Health, University of Toronto, Toronto, Canada \\ ${ }^{2}$ Genetic \& Genome Biology Department, SickKids Research Institute, Toronto, Canada \\ E-mail: jiamin.guo@mail.utoronto.ca
}

Received May 15, 2020

Accepted for publication June 1, 2020

Published online September 26, 2021

\begin{abstract}
Introduction: Cumulated advanced glycation end products (AGEs) in the bloodstream and tissues contribute to the pathogenesis of diabetes complications. The skin intrinsic fluorescence (SIF) is a non-invasive measurement of dermal AGEs level using spectrometer, and it can be used as a biomarker in AGEs-related diseases. Previously, specific epigenomic factor has been found to be associated with haemoglobin A1c (HbA1c). HbA1c is a type of glycated haemoglobin - the HbA1c test measures the average glycemic control over the period of 3 months. However, the effect of epigenetic factors on the level of AGEs in the skin remains unknown. We hypothesize that some cytosine-guanine dinucleotides (CpGs) are associated with SIF. An epigenome-wide associations study (EWAS) was performed to identify CpG sites associated with SIF in people with type 1 diabetes.
\end{abstract}

Methods: 499 people with type 1 diabetes that have both methylation and SIF from the Diabetes Control Complications Trial/Epidemiology of Diabetes Interventions and Complications (DCCT/EDIC) study were included. We fit linear regression models for SIF with each $\mathrm{CpG}$ site one at a time. The epigenome-wide significance level $(\mathrm{p}=5 \mathrm{e}-8)$ was applied. Then the result is compared with the null hypothesis where $\mathrm{CpGs}$ are not associated with SIF to check the inflation. In order to check the assumptions of the multiple linear models at a single $\mathrm{CpG}$, we use diagnostic plots.

Results: We did not identify a specific CpG that is significantly associated with neither skin intrinsic fluorescence 1 (SIF1) nor skin intrinsic fluorescence 12 (SIF 12). The CpG site with strongest effect is cg06538183 ([SE] -2.73 [0.61], p = 8.72e-6) on SIF1 and $\operatorname{cg} 12871967$ ([SE] 2.52, 0.53, p = 2.71e-6) on SIF12.

Conclusion: We did not find any specific $\mathrm{CpG}$ that was significantly associated with either SIF 1 or SIF12. In general, the result suggests that DNA methylation does not impact the accumulation of AGEs in skin cells. DNA methylation data has a unique pattern of distribution that drives the non-uniform distribution of the p-values. The group of 275,301 CpGs that have means above the median and standard deviations below the median has the expected uniform p-value distribution. 